\title{
Inhibition of growth hormone receptor by Somavert reduces expression of GPER and prevents growth stimulation of triple-negative breast cancer by $17 \beta$-estradiol
}

\author{
RAINER GIRGERT, GÜNTER EMONS and CARSTEN GRÜNDKER
}

\author{
Department of Obstetrics and Gynecology, University Medical Center Goettingen, D-37075 Goettingen, Germany
}

Received September 1, 2017; Accepted March 2, 2018

DOI: $10.3892 / \mathrm{ol} .2018 .8521$

\begin{abstract}
Currently, conventional chemotherapy is the only treatment option for triple-negative breast cancers (TNBC) due to a lack of a unique target. In TNBC, a high expression of the membrane bound $G$ protein-coupled estrogen receptor (GPER), correlates with a worse outcome. There is a potential for an association between growth hormone receptor (GHR) and GPER expression. To confirm this hypothesis, GHR was inhibited in TNBC cells with Somavert, and GPER expression levels, and the effect on signal transduction and proliferation induction in TNBC cells were analyzed. Proliferation of TNBC cells was measured using an Alamar-blue assay. Expression of GPER and activation of c-src and epidermal growth factor receptor (EGFR) by $17 \beta$-estradiol was analyzed by western blotting. Induction of c-fos, cyclin D1 and aromatase expression was determined by reverse transcription-semi-quantitative polymerase chain reaction. The expression of GPER was concentration- and time-dependently reduced by Somavert down to $46 \pm 7 \%(\mathrm{P}<0.01)$ of the control. Furthermore, 17 $\beta$-estradiol significantly increased the cell number of HCC1806 cells to $128 \pm 14 \%(\mathrm{P}<0.05)$, and that of MDA-MB-453 cells to $115 \pm 3 \%$. This increase in cell number was reduced to $103 \pm 11 \%$ in HCC1806 cells in which GPER expression was downregulated by Somavert, and to $102 \pm 3 \%$ in MDA-MB-453 cells. In addition, $17 \beta$-estradiol increased the activation of c-src in HCC1806 cells by 1.8 -fold, and Somavert reduced p-src to $63 \%$ of control. In MDA-MB-453 cells src phosphorylation increased by 7-fold upon stimulation with estradiol, but after treatment with Somavert only a 4-fold increase was observed. Phosphorylation of EGFR was increased by 2.2-fold of control in HCC1806 cells by $17 \beta$-estradiol, and by 1.4 -fold in MDA-MD-453 cells. Somavert completely prevented this activation. Induction of cyclin D1 and aromatase expression by $17 \beta$-estradiol was
\end{abstract}

Correspondence to: Dr Rainer Girgert, Department of Obstetrics and Gynecology, University Medical Center Goettingen, 40 Robert-Koch-Strasse, D-37075 Goettingen, Germany

E-mail: rainer.girgert@med.uni-goettingen.de

Key words: triple-negative breast cancer, targeted therapy, GPER, growth hormone receptor, Somavert, signal transduction also prevented by Somavert. Somavert reduces GPER expression in triple negative breast cancer cells. Treatment with Somavert prevents induction of genes regulating proliferation by $17 \beta$-estradiol. Inhibition of GPER expression is a promising therapeutic intervention for TNBC.

\section{Introduction}

Breast cancer is the most frequent malignant disorder in women. Patients with ER $\alpha$-positive tumors are amenable for therapy with tamoxifen and achieve an overall survival of approximately $81.3 \%$ after 5 years (1). But $10-15 \%$ of all breast cancer cases are designated triple-negative breast cancer (TNBC) as they neither expresses estrogen receptor ER $\alpha$ nor progesterone receptors and they do not overexpress Her-2. As a consequence, there is no successful targeted therapy available for TNBC patients and mortality of patients with TNBC is twice as high as for patients with ER $\alpha$ positive tumors (2). Mutations of the BRCA1 gene were identified as possible therapeutic target in TNBC, making these tumors particularly sensitive to platinum-compounds (3). In addition, the receptor for epidermal growth factor receptor (EGFR) is overexpressed in $30-52 \%$ of TNBC (4). A combination of anti-EGFR antibody Cetuximab and platinum compounds in the treatment of TNBC increased overall survival from 9.4 to 12.9 month (5).

A most recently discovered candidate for targeted therapy of TNBC is the membrane-bound estrogen receptor, $\mathrm{G}$ protein-coupled estrogen receptor (GPER). This heterotrimeric G-protein coupled receptor has a lower affinity for $17 \beta$-estradiol and is responsible for its nongenomic effects in various tissues. GPER mediates estrogen-induced signaling and proliferation in human breast epithelial cells and normal and malignant breast (6). An immunohistochemical analysis of tissue sections from TNBC tumors revealed a positive staining of $94 \%$ of TNBC samples. In particular, TNBC with high GPER expression was associated with younger age of patients. Recurrence rate of GPER-positive tumors was essentially higher than in a GPER-negative cohort (7). Our recent observation, that $17 \beta$-estradiol stimulates growth of TNBC cell lines, despite the lack of ER $\alpha$ expression, points to an involvement of GPER in malignant transformation of TNBC. A knock-down of GPER using specific siRNA completely prevented this growth stimulation of TNBC by $17 \beta$-estradiol (8). A pharmacological inhibition of GPER by the specifically 
developed inhibitor (G15) or with estriol was also successful in TNBC cell lines but super-physiologically high concentrations of these compounds were needed to achieve a sufficient inhibition of growth (9).

Alternatively, instead of a pharmacological inhibition of GPER, a reduction of GPER expression would lead to a lower activation of signaling pathways depending on GPER. A number of factors are established regulating GPER expression. Expression of GPER has been reported to correlate with over-expression of the receptor for EGF (10). In approximately $50 \%$ of TNBC cases EGFR was strongly expressed predicting short survival of patients carrying triple negative breast tumors (11). We have recently analyzed the impact of the tyrosine-kinase inhibitor gefitinib on the expression of GPER in TNBC cell lines. Treatment of TNBC cell lines HCC1806 and HCC70 with $200 \mathrm{nM}$ gefitinib for four days reduced GPER expression by $70 \%$. Activation of c-src and EGFR by $17 \beta$-estradiol was almost completely prevented in cells pretreated with gefitinib (12).

The growth hormone (GH)/insulin-like growth factor axis has been implicated in breast cancer progression and growth of MCF-7 xenografts was successfully prevented by the growth hormone receptor (GHR) antagonist Somavert (pegvisomant) (13). In addition, antagonists of GH-releasing hormone were shown to suppress in vivo growth of TNBC (14). This fact led us to the assumption that $\mathrm{GH}$ is a further factor involved in the regulation of GPER expression. To our knowledge the impact of a direct inhibition of GH-receptor on the expression of GPER has not yet been analyzed. Somavert (Pegvisomant) is a specific inhibitor of GH-receptor. It is a peptide of 191 amino acids with sequence-homology to GH. Solely, amino acid $\mathrm{Gly}_{120}$ is substituted in the original sequence by Lys or Arg and the peptide is chemically modified by the addition of PEG at five positions to increase solubility and stability of the compound (15).

Somavert has already been clinically applied for several years in treatment of acromegaly, a disease, caused in most cases by a pituitary adenoma leading to an over-production of GH responsible for the clinical features of acromegaly (16).

According to the above mentioned facts, it is plausible that reducing transcription of GPER by inhibition of the GHR is a promising procedure for the prevention of $17 \beta$-estradiol dependent growth stimulation of TNBC. In this study we analyzed whether expression of GPER in TNBC cell lines is down-regulated following inhibition of GHR using Somavert as competitive inhibitor. After reduction of GPER expression in TNBC cells using Somavert the consequences of this inhibition on the signaling of GPER were analyzed and the impact of the reduced GPER expression on the induction of proliferation by $17 \beta$-estradiol was measured. Since inhibition of GPER was shown to suppress expression of CCN family member 1 (CCN1; cysteine-rich angiogenic inducer 61, CYR61), a factor involved in tumor cell invasion (17), we also analyzed the impact of GPER downregulation by Somavert on expression of CCN1.

\section{Materials and methods}

Reagents. Somavert ${ }^{\mathrm{TM}}$ (Pegvisomant) was a generous gift from Pfizer (New York, NY, USA). 17ß-estradiol (E2), insulin and transferrin were purchased from Sigma-Aldrich; Merck KGaA (Darmstadt, Germany).
Cell lines. TNBC cell lines HCC1806, HCC70 and MDA-MB-453 were purchased from ATCC (Manassas, VA, USA) and maintained in DMEM containing $10 \%$ fetal bovine serum (both Biochrom, Berlin, Germany), supplemented with $2 \mathrm{mM}$ glutamine, $6 \mathrm{ng} / \mathrm{ml}$ insulin, $10 \mathrm{ng} / \mathrm{ml}$ transferrin, penicillin $(50 \mathrm{U} / \mathrm{ml})$, streptomycin $(50 \mu \mathrm{g} / \mathrm{ml})$ from Gibco; Thermo Fisher Scientific, Inc. (Paisley, UK).

Treatment of cells. To analyze the effect of Somavert on expression of GPER, four million cells of each cell line were grown in $2 \mathrm{ml}$ DMEM in $25 \mathrm{ml}$ tissue flasks. Cells were either treated with $1 \mu \mathrm{M}$ Somavert, the concentration clinically applied in treatment of acromegaly, for 48 or $96 \mathrm{~h}$.

For analysis of the impact of Somavert treatment on signal transduction of $17 \beta$-estradiol in TNBC cells, culture medium was replaced by phenolred-free culture medium without serum $24 \mathrm{~h}$ before stimulation of the cells with $10^{-8} \mathrm{M} 17 \beta$-estradiol for $15 \mathrm{~min}$. Cells were harvested in $1 \mathrm{mM}$ EDTA/PBS, centrifuged at $400 \mathrm{x}$ g and lysed in $50 \mu 1$ Cell Lytic M supplemented with protease- and phosphatase-inhibitors (Sigma-Aldrich; Merck KGaA).

Western blots. Lysates of cells were centrifuged at $15,000 \mathrm{x} \mathrm{g}$ for $5 \mathrm{~min}$ and protein concentration was measured using the method of Bradford. $20 \mu \mathrm{g}$ of each sample were loaded on a $7.5 \%$ polyacrylamide gel, run for one hour at $100 \mathrm{~V}$. Proteins were blotted on PVDF-membrane and sequentially detected with a series of rabbit primary antibodies: Anti-phospho-Src and anti-c-Src both from Cell Signaling Technology, Inc. (Danvers, MA, USA), anti-phospho Tyr ${ }^{173}$ EGFR delivered by Calbiochem (Darmstadt, Germany), anti-EGFR antibody from Epitomics (Hamburg, Germany) and anti-actin from Sigma-Aldrich; Merck KGaA. After four washes in TBST, blots were incubated with a $1: 20,000$ dilution of goat-anti-rabbit antibody conjugated with horseradish peroxidase (ECL; GE Healthcare Europe, GmbH, Freiburg, Germany). After further washing, blots were incubated with chemoluminescence reagent Femto (Thermo Fisher Scientific, Inc.) for $5 \mathrm{~min}$ and protein bands were detected on a LiCor chemoluminescence detector (Licor, Lincoln, NE, USA). Densitometric evaluation of the blots was performed with Image Studio Digits program from Licor. Expression values of the detected proteins were normalized to actin.

Proliferation assays. The proliferation assays for $17 \beta$-estradiol were performed in phenolred-free medium supplemented with charcoal depleted serum as previously described (18).

$10^{3}$ cells seeded in $100 \mu \mathrm{l}$ phenolred-free MEM supplemented with $2 \%$ charcoal depleted serum into 96 -well plates. Somavert was added in $50 \mu \mathrm{l}$ to achieve a final concentration of $1 \mu \mathrm{M}$. For stimulation $50 \mu \mathrm{l}$ of either vehicle or $4 \times 10^{-8} \mathrm{M}$ $17 \beta$-estradiol were added to four replicates.

Cells were grown for 7 days at $37^{\circ} \mathrm{C}, 5 \% \mathrm{CO}_{2}$ and saturated humidity. Cell number was determined by a colorimetric method as previously described (18).

Proliferation assays were performed at least three times with different passages. Means and standard deviations of the optical density (OD) of the replicates were calculated. 
Reverse transcription-semi-quantitative polymerase chain reaction ( $R T$-sqPCRs). RNA was purified from TNBC cells after pretreatment with $1 \mu \mathrm{M}$ Somavert and stimulation with $17 \beta$-estradiol using RNeasy-kit (Qiagen, Hilden, Germany).

Reverse transcription polymerase chain reaction was performed as previously described (8).

PCR-products were separated in a $2 \%$ agarose gel (Type IV, special high EEO; Sigma-Aldrich; Merck KGaA) and ethidium bromide stained gels were photographed using a CDS camera (Biometra, Göttingen, Germany).

Densitometric evaluation of PCR-products. The band intensities of the PCR-products were evaluated by the Digital science 1D-software (Kodak, Rochester, NY, USA). Values of the RT-PCR products were normalized to the ribosomal protein L7.

Statistical analysis. The data were tested for significant differences by one-way analysis of variance using GraphPad Prism 6.01-Software (GraphPad Software, Inc., La Jolla, CA, USA) followed by Student-Newman-Keuls'test for comparison of individual groups, after a Bartlett test had shown that variances were homogenous.

\section{Results}

Expression of GHR in TNBC. Expression of GHR of three TNBC-cell lines (HCC1806, HCC70 and MDA-MB-453) was compared to expression in ER $\alpha$-positive breast cancer cell line MCF-7 on western blotting. GHR was expressed strongest in HCC1806 and MDA-MB-453. HCC1806 cells expressed $118 \pm 24 \%$ and MDA-MB-453 cells $136 \pm 18 \%$ of the amount of GHR expressed in MCF-7. HCC70 cells contained exceptionally low amounts of GHR, approximately $2.4 \pm 0.8 \%(\mathrm{P}<0.01)$ of the amount detected in HCC1806 cells.

GPER expression was also highest in MDA-MB-453 cells. The amount of GPER detected in MDA-MB-453 was approximately $133 \pm 22 \%$ of the amount expressed in MCF-7 cells. GPER expression was lowest in HCC70 cells, being only $7.5 \pm 0.3 \%$ of GPER amount detected in MDA-MB-453 and correlated with the minimal amount of growth-hormone receptor found in this cell line (Fig. 1, lane 2). These results suggest that GPER expression might be regulated depending on GH-receptor.

Inhibition of growth-hormone receptor by Somavert reduces GPER expression. To prove this hypothesis the impact of inhibition of GH-receptor by the specific $\mathrm{GH}$-antagonist Somavert on GPER expression was analyzed in the TNBC cell lines. GPER expression was lowered concentration-dependently. As we have previously show for the reduction of GPER expression after inhibition of EGFR in HCC1806 using $500 \mathrm{nM}$ gefitinib, $96 \mathrm{~h}$ of treatment were needed to achieve maximally a reduction to $26 \pm 18 \%$ (12). To analyze the effect of an inhibition of GH-receptor on GPER expression the impact of various concentrations of Somavert were analyzed after $96 \mathrm{~h}$ of treatment. TNBC cells were treated with increasing concentrations $(0.25-1 \mu \mathrm{M})$ of Somavert for $96 \mathrm{~h}$. GPER expression was determined on western blotting of cellular lysates. In MDA-MB-453 cells,

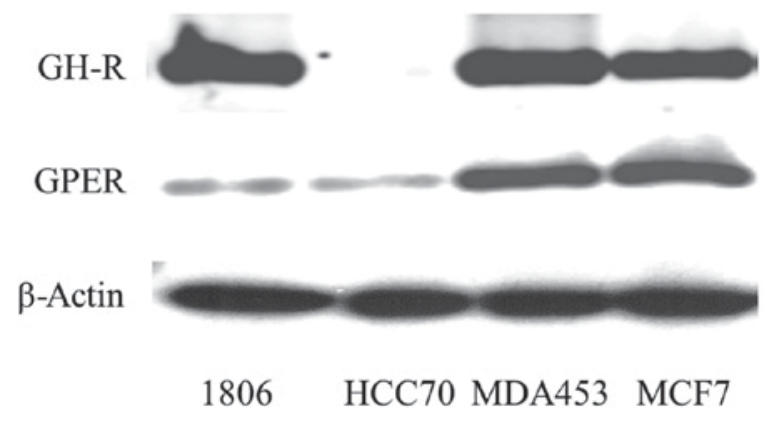

Figure 1. Expression of growth-hormone receptor and G protein-coupled estrogen receptor (GPER) in triple negative breast cancer cell lines. western blotting of cell lysates from triple-negative breast cancers (TNBC) cell lines HCC1806, HCC70 MDA-MB-453 and an ER $\alpha$-positive control (MCF-7) were sequentially detected with antibodies to the growth hormone receptor, to GPER and $\beta$-actin. $40 \mu \mathrm{g}$ protein of each cell lines were separated in a polyacrylamide-gel and transferred to a PVDF membrane. Representative western blotting of three independent experiments.

maximal reduction of GPER expression to $46 \pm 7 \%$ of control $(\mathrm{P}<0.05)$ was observed after treatment with $1 \mu \mathrm{M}$ Somavert for $96 \mathrm{~h}$. To show the inhibitory effect of Somavert on GPER expression in HCC1806 and HCC70 cells, expressing only low amounts of GPER according to Fig. 1, $100 \mu \mathrm{g}$ of cell lysates were loaded on Western blots to get a sufficient signal. In HCC1806 treated with $1 \mu \mathrm{M}$ for $96 \mathrm{~h}$ GPER expression was lowered to $56 \pm 5 \%(\mathrm{P}<0.01)$ of the amount detected in non-treated cells. In a third TNBC cell line, and in HCC70 cells GPER expression reached $58 \pm 6 \%(\mathrm{P}<0.05)$ of untreated control cells under these conditions (Fig. 2).

Somavert prevents stimulation of cell proliferation by $17 \beta$-estradiol in TNBC cells. Due to the lower affinity of GPER to $17 \beta$-estradiol compared to ER $\alpha$ the induction of proliferation of TNBC cell lines was compared at $10^{-8} \mathrm{M} 17 \beta$-estradiol in the absence or in the presence of $1 \mu \mathrm{M}$ Somavert (Fig. 3).

Stimulation of cell growth of HCC1806 cells by $10^{-8} \mathrm{M}$ $17 \beta$-estradiol increased cell number within 7 days of culture to $130 \pm 26 \%(\mathrm{P}<0.05)$ of controls. As reported earlier $(8)$ this growth stimulation is dependent on the presence of GPER in TNBC. $10^{-8} \mathrm{M} 17 \beta$-estradiol were necessary for maximal stimulation of GPER (9). If HCC1806 cells were additionally treated with $1 \mu \mathrm{M}$ Somavert the increase of cell number by $17 \beta$-estradiol was prevented and cell number remained at $103 \pm 11 \% \cdot 10^{-8} \mathrm{M} 17 \beta$-estradiol increased proliferation of MDA-MB-453 cells by $15 \%$ and cotreatment with $1 \mu \mathrm{M}$ Somavert completely prevented the effects of $17 \beta$-estradiol on cell number. In HCC70 cells an increase of cell number to $120 \pm 9 \%(\mathrm{P}<0.05)$ was achieved by $10^{-8} \mathrm{M} 17 \beta$-estradiol and after co-treatment with $1 \mu \mathrm{M}$ Somavert cell number still increased to $111 \pm 5 \%$ after 7 days of treatment (Fig. 3). This failure of Somavert to totally prevent induction of proliferation is in agreement with the smaller reduction of GPER expression due to lower amount of GHR in HCC70 cells (Fig. 2).

Reduction of GPER expression by Somavert inhibits phosphorylation of c-src and activation of EGFR by $17 \beta$-estradiol. Following stimulation of GPER with $17 \beta$-estradiol c-src and 
MDA-MB-453
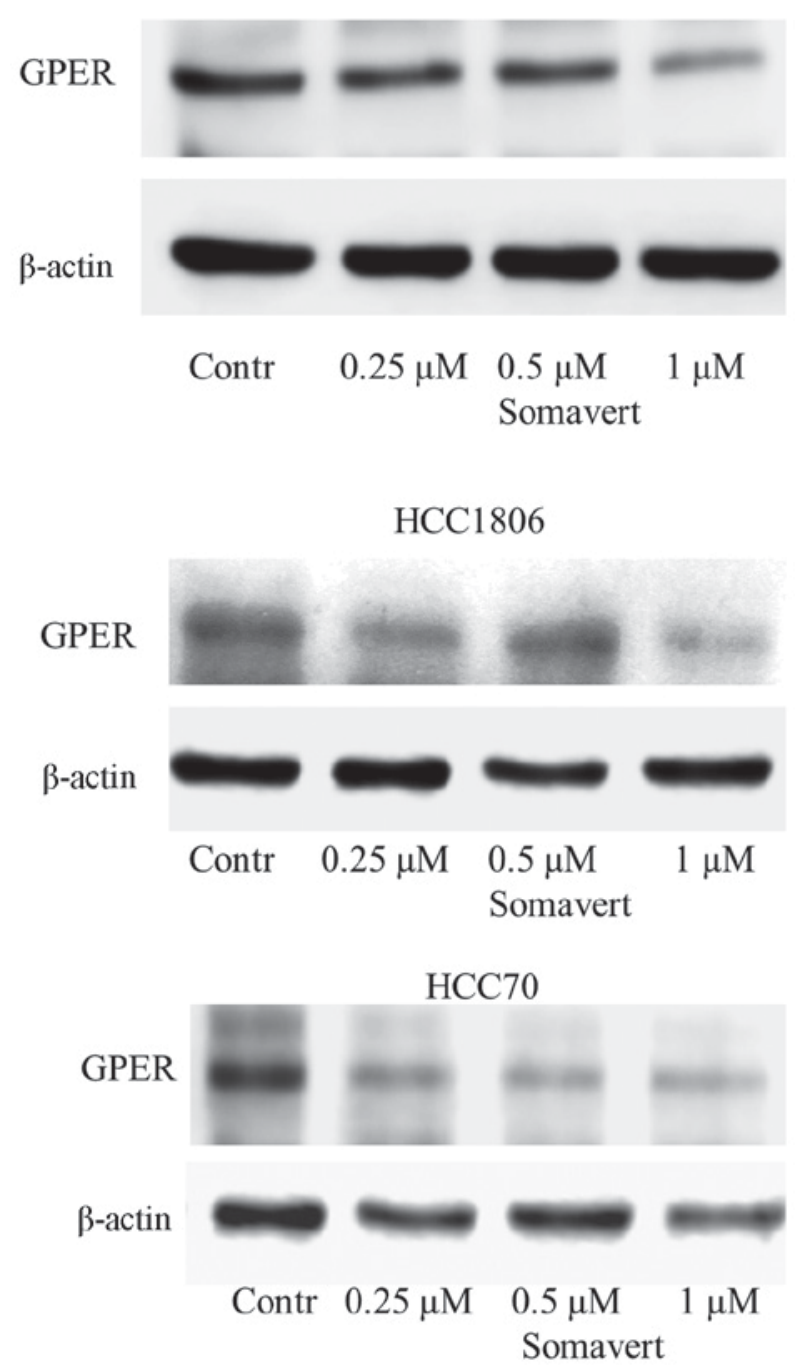

Figure 2. Reduction of G protein-coupled estrogen receptor (GPER) expression after treatment with Somavert. Cells of three triple-negative breast cancers (TNBC) cell lines were treated with increasing concentrations of Somavert for $96 \mathrm{~h}$. Western blotting of cell lysates (MDA-MB-453 $=40 \mu \mathrm{g}$; $\mathrm{HCC} 70$ and $\mathrm{HCC} 1806=100 \mu \mathrm{g}$ ) were detected with antibodies against GPER and actin. Representative results of three independent experiments.

EGFR are activated by phosphorylation $(8,19)$. As reported earlier (8) this growth stimulation is dependent on the presence of GPER in TNBC. Next, we analyzed activation of c-src and EGFR by $10^{-8} \mathrm{M} 17 \beta$-estradiol in TNBC cells after GPER expression was reduced after pretreatment by Somavert. Cells of the TNBC cell lines, HCC1806, HCC70 and MDA-MB453 were treated with $1 \mu \mathrm{M}$ Somavert for four days and stimulated with 17ß-estradiol. Phosphorylation of c-src and EGFR was compared to control cells, not treated with Somavert on western blots (Fig. 4).

In serum starved MDA-MB-453 cells phosphorylation of c-src at $\mathrm{Tyr}^{416}$ was very weak. A 15 min stimulation with $10^{-8} \mathrm{M} 17 \beta$-estradiol lead to a 7.3 -fold increase of c-src phosphorylation. After pretreatment of the cells with $1 \mu \mathrm{M}$ Somavert, $17 \beta$-estradiol only led to a 4 -fold activation of c-src. Even in the serum-starved TNBC cells of HCC70 and HCC1806 a basal phosphorylation of c-src was already quite strong (Fig. 4, lane 1). In HCC1806 cells the activation of c-src

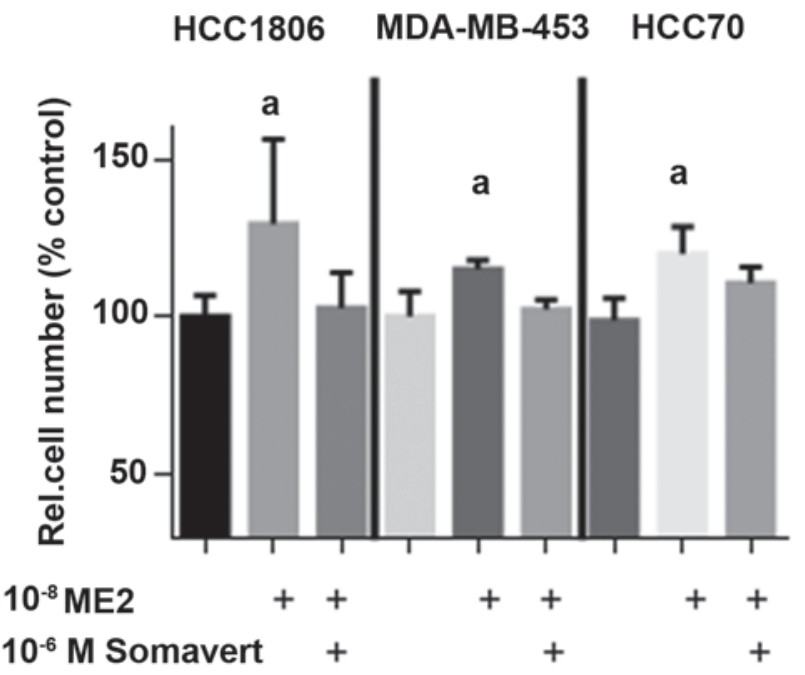

Figure 3. Inhibition of $17 \beta$-estradiol induced cell proliferation by Somavert in triple-negative breast cancers (TNBC) cell lines. Cells were grown for 7 days in phenolred-free medium supplemented with charcoal-stripped serum (control) or in the presence of $10^{-8} \mathrm{M} 17 \beta$-estradiol or cotreated with $10^{-6} \mathrm{M}$ Somavert. Cell number was estimated with a colorimetric assay using Alamar blue. Mean values and SE of three independent experiments with four replicates. ${ }^{\mathrm{a}} \mathrm{P}<0.0510^{-8} \mathrm{M} \mathrm{E} 2 \mathrm{vs}$. negative control.

by the treatment with $17 \beta$-estradiol amounted to $177 \pm 49 \%$ $(\mathrm{P}<0.01$; Fig. 4, lane 2). A stimulation of $\mathrm{HCC} 70$ cells with $10^{-8} \mathrm{M} 17 \beta$-estradiol lead to an increase of $\mathrm{p}$-src to $125 \pm 32 \%$ of control $(\mathrm{P}<0.05)$. In MDA-MB-453 cells and HCC1806 cells expressing high amounts of GHR the reduction of GPER expression following treatment with $1 \mu \mathrm{M}$ Somavert for $96 \mathrm{~h}$ prevented the increase of c-src phosphorylation by 173-estradiol (Fig. 4, lane 4). In HCC70 cells wherein GPER expression was less strongly reduced by Somavert treatment $17 \beta$-estradiol was still able to induce c-src phosphorylation although weaker than in non-treated HCC70 cells to $110 \pm 9 \%$ (P<0.05; Fig. 4, lane 4).

Phosphorylation of src subsequently leads to release of heparin-bound EGF from extracellular matrix by matrixmetalloproteases. The released EGF initiates autophosphorylation of the cytosolic domain of the EGFR. In all three TNBC cell lines $\mathrm{Tyr}^{1173}$ phosphorylation of the EGFR was analyzed after stimulation with $10^{-8}$ M $17 \beta$-estradiol (Fig. 4, lane 2). In MDA-MB-453 cells phosphorylation of EGFR increased to $136 \pm 19 \%$ of non-stimulated control $(\mathrm{P}<0.05)$. Stimulation of $\mathrm{HCC} 1806$ cells elevated p-EGFR level to $216 \pm 24 \%$ of control $(\mathrm{P}<0.01)$ and in HCC70 cells to $202 \pm 112 \%$ ( $\mathrm{P}<0.05$; Fig. 4, lane 2 ).

In MDA-MB-453- and HCC1806-cells phosphorylation of $\mathrm{Tyr}^{1173}$ by $17 \beta$-estradiol was completely prevented in cells pretreated with $1 \mu \mathrm{M}$ Somavert (Fig. 4, lane 4). But in HCC70 cells expressing lower amounts of GHR Somavert was less effective in preventing the induction of EGFR phosphorylation. In HCC70 cells pretreated with $1 \mu \mathrm{M}$ Somavert $17 \beta$-estradiol was still able to increase EGFR phosphorylation to $166 \pm 59 \%$ of control $(\mathrm{P}<0.05$; Fig. 4 , lane 4$)$. This observation provides further evidence that GPER expression is regulated at least in part by the GHR.

Somavert inhibits induction of cyclin D1 expression by $17 \beta$-estradiol. Cyclin D1 regulates of the transition from 


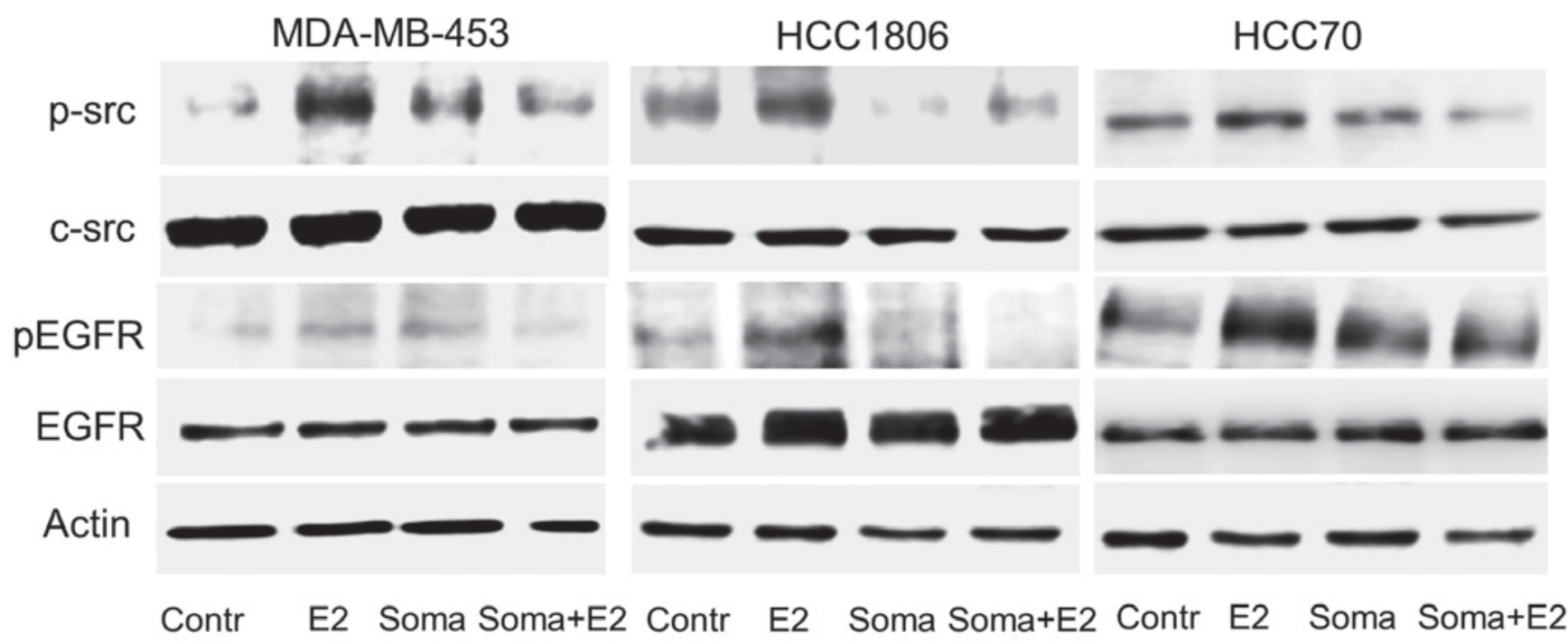

Figure 4. Inhibition of G protein-coupled estrogen receptor (GPER) dependent signal transduction in three different triple-negative breast cancers (TNBC) cell lines after treatment with Somavert. Somavert prevents src activation by $17 \beta$-estradiol. Somavert prevents epidermal growth factor receptor (EGFR) activation by $17 \beta$-estradiol. Serum starved cells (control) were stimulated for ten min with $10^{-8} \mathrm{M} 17 \beta$-estradiol (lane 2) or pretreated for $96 \mathrm{~h}$ with $1 \mu \mathrm{M}$ Somavert (lane 3) and Somavert treated cells were subsequently stimulated with $10^{-8} \mathrm{M} 17 \beta$-estradiol for ten min (lane 4). Cells were lysed, proteins separated in a polyacrylamide gel, blotted onto a PVDF-membrane, and the indicated proteins were sequentially detected with antibodies against phospho-src or total-src antibodies against phospho-EGFR or total EGFR. Representative results of three independent preparations.

G1-phase to S-phase of the cell cycle. As we reported earlier expression of cyclin D1 and aromatase is induced by $17 \beta$-estradiol in a GPER dependent manner (8). Expression of cyclin D1 was analyzed in Somavert treated TNBC cells by RT-PCR after stimulation with $10^{-8} \mathrm{M} 17 \beta$-estradiol (Fig. 5, first panel).

In HCC1806 cells expression of cyclin D1 significantly increased to $116 \pm 11 \%$ of control $(\mathrm{P}<0.05)$ after $30 \mathrm{~min}$ stimulation with $17 \beta$-estradiol. In HCC1806 cells treated with Somavert cyclin D1 expression did not increase after stimulation with 17ß-estradiol for $30 \mathrm{~min}$ (Fig. 5C).

In control cells of MDA-MB-453 cyclin D1 was more strongly expressed than in HCC1806 (Fig. 5B, lane 1). Stimulation of these cells with $17 \beta$-estradiol increased cyclin D1 expression only to $117 \pm 29 \%$ of control. Pretreatment of MDA-MB-453 cells with $1 \mu \mathrm{M}$ Somavert completely prevented induction of cyclin D1 expression by $17 \beta$-estradiol (Fig. 5E).

Aromatase expression in TNBC cells. The aromatase gene essential for an autocrine biosynthesis of $17 \beta$-estradiol in TNBC is upregulated via GPER (9). Stimulation of HCC1806 cells with $17 \beta$-estradiol increased aromatase expression to $131 \pm 26 \%$ of control $(\mathrm{P}<0.05)$. Reduction of GPER expression by Somavert reduced the induction of aromatase expression by $17 \beta$-estradiol only marginally to $121 \pm 51 \%$ (Fig. 5D). In MDA-MB-453 cells $17 \beta$-estradiol increased aromatase expression only to $110 \pm 36 \%$ of control. If MDA-MB- 453 cells were pretreated with $10^{-6} \mathrm{M}$ Somavert for $96 \mathrm{~h}$ induction of aromatase expression was lowered below control level (Fig. 5F).

CCN1 expression is not induced by $17 \beta$-estradiol nor is it reduced by Somavert treatment. CCN1 (Cyr61) is a secreted protein that plays divers roles in cellular proliferation, survival and migration (17). Estrogen is a powerful inducer of CCN1 in breast cancer cells. In order to prove, whether CCN1 induction in TNBC cells is dependent on expression of GPER, expression of CCN1 was analyzed after stimulation with $17 \beta$-estradiol in TNBC cells with and without pretreatment with Somavert (data not shown). In HCC1806- and MDA-MB-453 cells $17 \beta$-estradiol was not able to increase $\mathrm{CCN} 1$ expression, neither on mRNA level nor on protein level (data not shown).

\section{Discussion}

Triple negative breast tumors lack of the expression of estrogen receptor $\alpha(E R \alpha)$ and of progesterone receptors and do not overexpress Her-2, the target of Trastuzumab. Therefore, therapies using the antiestrogen Tamoxifen or antibody therapy with Trastuzumab are no rationale. GPER, a membrane-bound receptor for estrogens, initiates fast non-genomic effects of $17 \beta$-estradiol that are independent of $\mathrm{ER} \alpha$. GPER might become a promising target in treatment of TNBC. Most tumors of TNBCs were shown to express GPER by immunohistochemical staining. High GPER expression predicted a higher recurrence rate of TNBC (7). Therefore, we assume that stimulation of GPER by circulating $17 \beta$-estradiol contributes to the malignant behavior of TNBC. We were already able to show that $17 \beta$-estradiol increases proliferation of TNBC cells $(8,9,12)$. On the other hand, antiestrogens, like tamoxifen or fulvestrant, that inhibit $\mathrm{ER} \alpha$, are agonists of GPER and therefor these antiestrogens are not clinically applicable against triple negative breast tumors (20). 
A
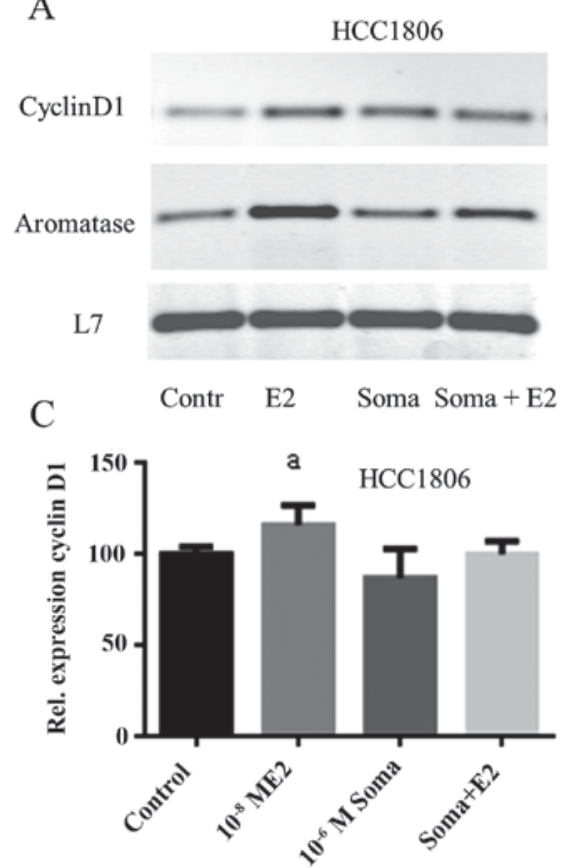

E

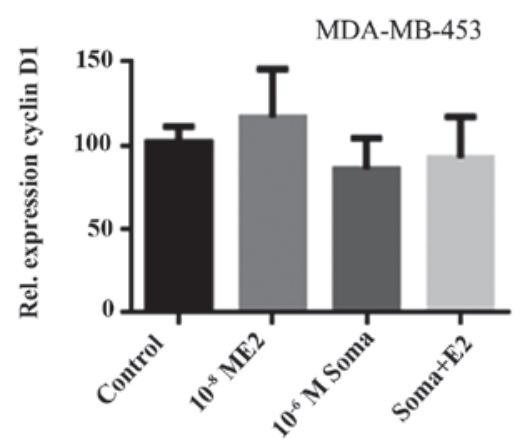

B

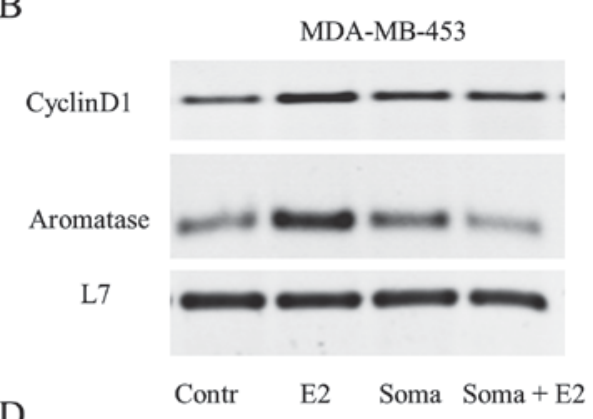

$\mathrm{D}$

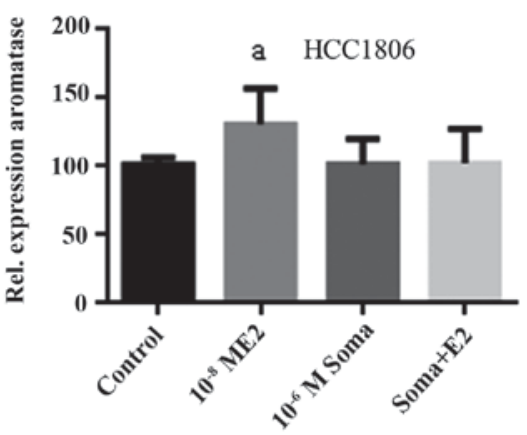

F

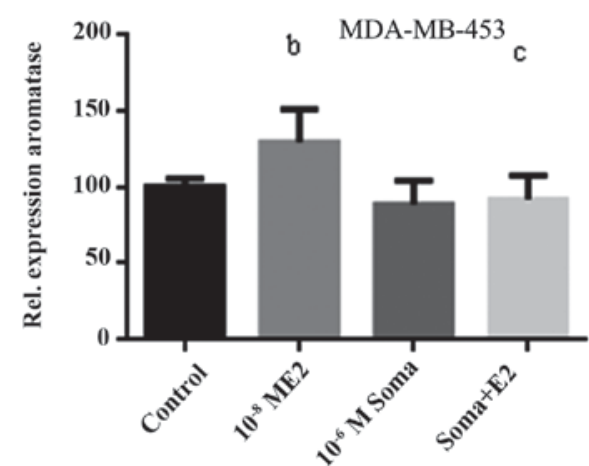

Figure 5. Influence of Somavert on the induction of cyclin D1-, and aromatase-expression by $17 \beta$-estradiol in TNBC cell lines. Cells of HCC1806 and MDA-MB-453 were grown in serum-free medium for $24 \mathrm{~h}$ (Control) and subsequently stimulated with $10^{-8} \mathrm{M} 17 \beta$-estradiol for $30 \mathrm{~min}$ (E2). Cells were pretreated for $96 \mathrm{~h}$ with $10^{-6} \mathrm{M}$ Somavert and serum starved for $24 \mathrm{~h}$ (Soma) or subsequently stimulated with $17 \beta$-estradiol for $30 \mathrm{~min}$ (Soma+E2). RT-PCR for cyclin D1 and aromatase (CYP19A1) was performed with mRNA isolated from the variously treated cells. RT-PCR for the ribosomal protein L7 proves equal RNA loading. (A) HCC1806 Representative RT-PCR-products of cyclin D1, aromatase and L7 mRNA (n=3). (B) MDA-MB-453 Representative RT-PCR-products of cyclin D1, aromatase and L7 mRNA ( $\mathrm{n}=3$ ). (C) Densitometric analysis of cyclin D1-PCR-product from HCC1806. (D) Densitometric analysis of aromatase PCR-products from HCC1806. (E) Densitometric analysis of cyclin D1-PCR-products from MDA-MB-453. (F) Densitometric analysis of aromatase-PCR-products from MDA-MB-453. ${ }^{a} \mathrm{P}<0.05$ vs. control; ${ }^{\mathrm{b}} \mathrm{P}<0.01$ vs. control; ${ }^{\mathrm{c}} \mathrm{P}<0.05 \mathrm{vs} .10^{-8} \mathrm{M}$ E2.

A pharmacological inhibition of GPER by estriol and G15, a compound selective for GPER, inhibited signal transduction of GPER and reduced proliferation of TNBC cells only at extremely high non-physiological concentrations (9).

Due to this disadvantage we decided to follow a different path to prevent growth stimulation of TNBC cells by $17 \beta$-estradiol via GPER. A reduction of GPER expression might have similar effects as pharmacological inhibition of GPER. It has been shown, that in TNBC expression of GPER correlates with expression of EGFR (10). Inhibition of EGFR with tyrosine-kinase inhibitor gefitinib reduced GPER expression by up to $85 \%$ (12).

Recently, Perez et al reported that growth of TNBC tumors is stimulated autocrinely by the GH releasing hormone (GHRH) (14). For this reason we analyzed in this report, whether GPER expression is regulated by the GH. A comparison of GPER expression and expression of GHR in a number of TNBC cell lines showed that in three of four cell lines GPER correlated with GHR. Somavert is a competitive inhibitor of the GHR. As further proof, that GPER is regulated by GH we observed that inhibition of GHR using $1 \mu \mathrm{M}$ Somavert, a concentration clinically applied in the treatment of acromegaly, led to a reduction of GPER expression in all TNBC cell lines tested for up to 54\%.

As a consequence, treatment of TNBC cell lines MDA-MB-453 and HCC1806 with $1 \mu \mathrm{M}$ Somavert for $96 \mathrm{~h}$ prevented the $17 \beta$-estradiol dependent activation of c-src completely. In the TNBC cell line HCC70 inhibition of c-src activation by Somavert was less pronounced than in the other two TNBC cell lines tested, probably due to the fact that this cell line expresses less GHR and GPER expression in this cell line was less sensitive to treatment with Somavert. This observation is in concert with our previous finding that in HCC70 cells GPER expression is more strongly dependent on stimulation of the EGFR (12). 
In the signaling pathway of GPER downstream of c-src the activation of EGFR following stimulation of the TNBC cell lines MDA-MB-453 and HCC1806 with 17 $\beta$-estradiol was also reduced in cells pretreated with Somavert. In contrast, in HCC70 cells activation of EGFR was also less strongly prevented by Somavert for the same reasons as described for c-src.

The induction of the genes relevant for proliferation, c-fos, and Cyclin D1, after stimulation of the TNBC cells with $17 \beta$-estradiol was subsequently prevented in the cells pretreated with Somavert. This observation additionally proves that reduction of GPER by Somavert leads to a specific downregulation of cell growth.

A further gene, reported to be regulated by $17 \beta$-estradiol in ER $\alpha$-negative breast cancer cells, is CCN1 (Cyr61). $\mathrm{CCN} 1$ plays divers roles in cellular proliferation, survival and migration. Estrogen has been shown to be an inducer of CCN1 in MDA-MB-231 breast cancer cells (17). However, in MDA-MB-453 and HCC1806 TNBC cells $17 \beta$-estradiol did not increase CCN1 expression.

A complex interaction between estrogens and $\mathrm{GH}$ in the regulation of breast cancer growth has formerly been described. In ovariectomized rats supplementation of estrogen increased the level of $\mathrm{GH}$ and increased expression of GHR at least on osteosarcoma and liver cells $(21,22)$. On the other hand, estrogens were shown to inhibit signaling of GH-receptors by suppressing GH-dependent JAK phosphorylation. This effect is exerted by induction of SOCS expression by estradiol, a known negative regulator of signaling of several cytokine receptors (23). But vice versa $\mathrm{GH}$ also influences effects of estrogens via GPER expression as shown in the present report.

Somavert is clinically applied for the treatment of acromegaly, a disorder observed in patients suffering from a tumor in the pituitary, secreting high amounts of GH (16). In patient suffering from acromegaly treatment with Somavert was experienced as effective and safe with only minor side effects (15). Therefore, application of Somavert in the treatment of patients with triple negative breast cancer would be of low risk.

\section{Acknowledgements}

The authors would like to thank Mrs Sonja Blume and Mr. Matthias Läsche for their technical assistance.

\section{Funding}

This study was supported by a grant from the German Research Foundation (grant no. GR 1895/10-1).

\section{Availability of data and materials}

The datasets used and/or analyzed during the current study are available from the corresponding author on reasonable request.

\section{Authors' contributions}

RG and CG together developed the conception of the project. RG carried out all experiments, performed data analysis and drafted the manuscript. CG participated in the design of the study and the statistical analysis and he supervised the drafting of the manuscript. GE critically revised the manuscript and approved the final version.

\section{Ethics approval and consent to participate}

Not applicable.

\section{Consent for publication}

Not applicable.

\section{Competing interests}

The authors declare that they have no competing interests.

\section{References}

1. Dowsett M, Cuzick J, Ingle J, Coates A, Forbes J, Bliss J, Buyse M, Baum M, Buzdar A, Colleoni M, et al: Meta-analysis of breast cancer outcomes in adjuvant trials of aromatase inhibitors versus tamoxifen. J Clin Oncol 28: 509-518, 2010.

2. Carey LA, Dees EC, Sawyer L, Gatti L, Moore DT, Collichio F, Ollila DW, Sartor CI, Graham ML and Perou CM: The triple negative paradox: Primary tumor chemosensitivity of breast cancer subtypes. Clin Cancer Res 13: 2329-2334, 2007.

3. Silver DP, Richardson AL, Eklund AC, Wang ZC, Szallasi Z, Li Q, Juul N, Leong CO, Calogrias D, Buraimoh A et al: Efficacy of neoadjuvant Cisplatin in triple-negative breast cancer. J Clin Oncol 28: 1145-1153, 2010.

4. Reis-Filho JS and Tutt AN: Triple negative tumours: A critical review. Histopathology 52: 108-118, 2008.

5. Baselga J, Gómez P, Greil R, Braga S, Climent MA, Wardley AM, Kaufman B, Stemmer SM, Pego A, Chan A, et al: Randomized phase II study of the anti-epidermal growth factor receptor monoclonal antibody cetuximab with cisplatin versus cisplatin alone in patients with metastatic triple-negative breast cancer. J Clin Oncol 31: 2586-2592, 2013.

6. Scaling AL, Prossnitz ER and Hathaway HJ: GPER mediates estrogen-induced signaling and proliferation in human breast epithelial cells and normal and malignant breast. Horm Cancer 5: 146-160, 2014.

7. Steiman J, Peralta EA, Louis S and Kamel O: Biology of the estrogen receptor, GPR 30, in triple negative breast cancer. Am J Surg 206: 698-703, 2013.

8. Girgert R, Emons G and Gründker C: Inactivation of GPR30 reduces growth of triple-negative breast cancer cells: Possible application in targeted therapy. Breast Cancer Res Treat 134: 199-205, 2012.

9. Girgert R, Emons G and Gründker C: Inhibition of GPR30 by estriol prevents growth stimulation of triple-negative breast cancer cells by $17 \beta$-estradiol. BMC Cancer 14: 935, 2014.

10. Vivacqua A, Lappano R, De Marco P, Sisci D, Aquila S, De Amicis F, Fuqua SA, Andó $S$ and Maggiolini M: G protein-coupled receptor 30 expression is up-regulated by EGF and TGF alpha in estrogen receptor alpha-positive cancer cells. Mol Endocrinol 23: 1815-1826, 2009.

11. Nielsen TO, Hsu FD, Jensen K, Cheang M, Karaca G, Hu Z, Hernandez-Boussard T, Livasy C, Cowan D, Dressler L, et al: Immunohistochemical and clinical characterization of the basal-like subtype of invasive breast carcinoma. Clin Cancer Res 10: 5367-5374, 2004.

12. Girgert R, Emons G and Gründker C: $17 \beta$-estradiol-induced growth of triple-negative breast cancer cells is prevented by the reduction of GPER expression after treatment with gefitinib. Oncol Rep 37: 1212-1218, 2017.

13. Divisova J, Kuiatse I, Lazard Z, Weiss H, Vreeland F, Hadsell DL, Schiff R, Osborne CK and Lee AV: The growth hormone receptor antagonist pegvisomant blocks both mammary gland development and MCF-7 breast cancer xenograft growth. Breast Cancer Res Treat 98: 315-327, 2006.

14. Perez R, Schally AV, Vidaurre I, Rincon R, Block NL and Rick FG: Antagonists of growth hormone-releasing hormone suppress in vivo tumor growth and gene expression in triple negative breast cancers. Oncotarget 3: 988-997, 2012. 
15. Neggers SJ, Franck SE, de Rooij FW, Dallenga AH, Poublon RM, Feelders RA, Janssen JA, Buchfelder M, Hofland LJ, Jorgensen $\mathrm{JO}$, et al: Long-term efficacy and safety of pegvisomant in combination with long-acting somatostatin analogs in acromegaly. J Clin Endocrinol Metab 99: 3644-3652, 2014.

16. Neggers SJ and van der Lely AJ: Somatostatin analog and pegvisomant combination therapy for acromegaly. Nat Rev Endocrinol 5: 546-552, 2009.

17. Kireeva ML, Mo FE, Yang GP and Lau LF: Cyr61, a product of a growth factor-inducible immediate-early gene, promotes cell proliferation, migration, and adhesion. Mol Cell Biol 16 : 1326-1334, 1996.

18. Girgert R, Bartsch C, Hill SM, Kreienberg R and Hanf V: Tracking the elusive antiestrogenic effect of melatonin: A new methodological approach. Neuro Endocrinol Lett 24: 440-444, 2003.

19. Filardo EJ, Quinn JA, Frackelton AR Jr and Bland KI: Estrogen action via the G protein-coupled receptor, GPR30: Stimulation of adenylyl cyclase and cAMP-mediated attenuation of the epidermal growth factor receptor-to-MAPK signaling axis. Mol Endocrinol 16: 70-84, 2002.
20. Ignatov A, Ignatov T, Weissenborn C, Eggemann $\mathrm{H}$, Bischoff J, Semczuk A, Roessner A, Costa SD and Kalinski T: G-protein-coupled estrogen receptor GPR30 and tamoxifen resistance in breast cancer. Breast Cancer Res Treat 128: 457-466, 2011.

21. Contreras B and Talamantes F: Growth hormone $(\mathrm{GH})$ and 17beta-estradiol regulation of the expression of mouse $\mathrm{GH}$ receptor and GH-binding protein in cultured mouse hepatocytes. Endocrinology 140: 4725-4731, 1999.

22. Slootweg MC, Swolin D, Netelenbos JC, Isaksson OG and Ohlsson C: Estrogen enhances growth hormone receptor expression and growth hormone action in rat osteosarcoma cells and human osteoblast-like cells. J Endocrinol 155: 159-164, 1997.

23. Birzniece V, Sata A and Ho KK: Growth hormone receptor modulators. Rev Endocr Metab Disord 10: 145-156, 2009. 